\title{
HIGH-ORDER LEGENDRE COLLOCATION METHOD FOR FRACTIONAL-ORDER LINEAR SEMI-EXPLICIT DIFFERENTIAL ALGEBRAIC EQUATIONS*
}

\author{
F. GHANBARI ${ }^{\dagger}$, K. GHANBARI ${ }^{\dagger}$, AND P. MOKHTARY ${ }^{\dagger}$
}

\begin{abstract}
This paper is devoted to a high-order Legendre collocation approximation for solving fractional-order linear semi-explicit differential algebraic equations numerically. We discuss existence, uniqueness, and regularity results and conclude that the solutions typically suffer from a singularity at the origin. Moreover, we show that the representation of the approximate solutions by a linear combination of Legendre polynomials leads to unsatisfactory convergence results. To overcome this difficulty, we develop a new regularization approach that removes the singularity of the input data and produces approximate solutions of higher accuracy. Illustrative numerical examples are presented to support the obtained theoretical results.
\end{abstract}

Key words. fractional-order differential algebraic equation, Legendre collocation method, regularization approach.

AMS subject classifications. 34A08, 65L05, 65L20, 65L60, 65L80.

1. Introduction. This work investigates a high-order Legendre collocation approach for approximating the solutions of the following fractional-order linear semi-explicit differential algebraic equation

$$
\left\{\begin{aligned}
D^{\alpha} x(t) & =p_{1}(t) x(t)+p_{2}(t) y(t)+q_{1}(t) \\
0 & =p_{3}(t) x(t)+p_{4}(t) y(t)+q_{2}(t) \\
x(0) & =d_{0}, \quad y(0)=d_{1}, \quad t \in I=[0,1],
\end{aligned}\right.
$$

where $\alpha=\frac{p}{q} \in \mathbb{Q} \cap(0,1)$ with two relatively prime integers $p \geq 1$ and $q \geq 2$. $\mathbb{Q}$ is the set of rational numbers, and the constants $d_{0}$ and $d_{1}$ are given. The known coefficient functions $p_{i}(t), q_{j}(t), i=1, \ldots, 4, j=1,2$, are continuous on $I$, and $D^{\alpha}$ is the Caputo-type fractional derivative operator of order $\alpha$ defined as (cf. [4, 12])

$$
D^{\alpha} x(t)=I^{1-\alpha}\left(x^{\prime}\right),
$$

where

$$
I^{\alpha} x(t)=\frac{1}{\Gamma(\alpha)} \int_{0}^{t}(t-s)^{\alpha-1} x(s) d s,
$$

is the Riemann-Liouville-type fractional integral operator of order $\alpha$ and $\Gamma(\alpha)$ denotes the Gamma function. Some of the properties of these operators are given in [4],

$$
I^{\alpha}\left(D^{\alpha} x(t)\right)=x(t)-x(0), \quad D^{\alpha} t^{k}= \begin{cases}\frac{k !}{\Gamma(k-\alpha+1)} t^{k-\alpha} & k \in \mathbb{N}, \\ 0 & k=0,\end{cases}
$$

where $\mathbb{N}$ is the set of natural numbers.

Recently, equations of this type came up in many important mathematical models such as electrochemical processes, non-integer-order optimal controller design and complex biochemical processes [3, 16], and circuit models containing super-conducting components [17, 18].

*Received May 12, 2017. Accepted September 2, 2018. Published online on November 16, 2018. Recommended by JaEun $\mathrm{Ku}$.

${ }^{\dagger}$ Department of Mathematics, Faculty of Basic Sciences, Sahand University of Technology, Tabriz, Iran (\{kghanbari, f_ghanbari, mokhtary\}@sut.ac.ir, mokhtary.payam@gmail.com). 


\section{ETNA}

Kent State University and

Johann Radon Institute (RICAM)

Sometimes one can convert fractional differential algebraic equations (FDAEs) into an equivalent fractional differential equations, and this can be useful in investigating theoretical aspects of the solution such as existence, uniqueness, and asymptotic performance of the solution. However, the numerical solution of the equivalent fractional differential equations instead of the original one may cause some crucial drawbacks. For example, since FDAEs exhibit a collection of relationships between variables of interest and usually some of these variables and their fractional-order derivatives have a physical significance, changing the model may generate less meaningful variables. Also, the numerical solution of the equivalent system no longer satisfies the original constraints exactly, and by the fact that the constraints reflect important physical properties, this could be a serious problem. In addition, from the numerical point of view, transforming FDAEs into fractional differential equations can increase the computational costs and destroy sparsity and prevent the exploitation of the structure of the system. Furthermore, by studying FDAEs directly, researchers can analyze more easily the effect of modeling variations, and linking the modeling software to the design software becomes simpler. Thus, in this paper we focus on obtaining a numerical solution by considering FDAEs directly [1].

In recent years, a few methods have been used to solve FDAEs numerically such as the generalized triangular function operational matrices method [3], the waveform relaxation method (WRM) [5], the fractional differential transform method (FDTM) [7], the variational iteration method (VIM), the Adomian decomposition method (ADM) [6], the Laplace homotopy analysis method (LHAM) [8], and the homotopy analysis method (HAM) [19]. But most of the techniques do not involve any convergence analysis and the reported numerical results indicate approximations of low accuracy even when the exact solution is sufficiently smooth. Thus, introducing and analyzing an approximate method which provides high accuracy for the numerical solution of (1.1) could be very important and new in the literature.

In this paper, we pursue the development of a suitable numerical approach for approximating the solutions of (1.1). To this end, we present a technique mainly consisting of three stages. First, we give an existence and uniqueness theorem along with a regularity result for (1.1) and prove that some derivatives of $x(t)$ and $y(t)$ have a singularity at the origin and behave like $t^{\alpha-1}$. Second, we adopt the Legendre collocation method for approximating (1.1). The obtained numerical results and theoretical predictions indicate that, due to the singularities of the derivatives of the exact solutions, this strategy yields numerical solutions with low accuracy. In the third step, for obtaining high-order approximate solutions, we proceed with a regularization approach using the asymptotic performance of the unknown solutions that allows us to improve the smoothness of the input functions and to approximate the solutions by means of a Legendre collocation method of higher accuracy.

The organization of this article is as follows: The existence and uniqueness theorem along with a regularity result for (1.1) are given in Section 2. In Section 3, we introduce and analyze the Legendre collocation scheme to approximate the solution of (1.1). In Section 4, we first change (1.1) into a new equation with higher regularity by means of a suitable smoothing procedure, and then the Legendre collocation solutions of the transformed equation are obtained. Moreover, the effects of the proposed regularization approach in producing highorder approximate solutions are justified. Some numerical examples are provided to confirm the obtained theoretical predictions in Section 5. The final section contains a conclusion.

2. Existence and uniqueness theorem. The main goal of this section is to demonstrate the existence and uniqueness of solutions of (1.1) and their regularity properties. To this end, it is necessary to recall the following theorem regarding the existence and uniqueness result for the solutions of fractional-order differential equations. 
TheOREM 2.1 (Theorems 6.5, 6.32, and Corollary 6.34 in [4]). Let $\alpha=\frac{p}{q} \in(0,1)$ with two relatively prime integers $p \geq 1$ and $q \geq 2$, and assume that the continuous function $f: I \times \mathbb{R} \rightarrow \mathbb{R}(\mathbb{R}$ denotes the set of real numbers) satisfies a Lipschitz condition with respect to the second variable, i.e.,

$$
\left|f\left(t, x_{1}(t)\right)-f\left(t, x_{2}(t)\right)\right| \leq C\left|x_{1}(t)-x_{2}(t)\right|,
$$

with some constant $C>0$ independent of $t, x_{1}, x_{2}$. Then, the fractional differential equation

$$
\left\{\begin{aligned}
D^{\alpha} x(t) & =f(t, x(t)) \\
x(0) & =d_{0}, \quad t \in I,
\end{aligned}\right.
$$

has a unique continuous solution $x(t)$ on $I$. Moreover, if the function $f$ can be written as $f(t, x(t))=\bar{f}\left(t^{\frac{1}{q}}, x(t)\right)$ with an analytic function $\bar{f}$ in a neighborhood of $\left(0, d_{0}\right)$, then there exists a unique analytic function $\bar{x}(t):(-r, r) \rightarrow \mathbb{R}$ with some $r>0$ such that

$$
x(t)=\bar{x}\left(t^{\frac{1}{q}}\right)=d_{0}+\sum_{i=p}^{\infty} \bar{x}_{i} t^{\frac{i}{q}}, \quad \text { with } \quad\left|x^{\prime}(t)\right| \leq C_{\alpha} t^{\alpha-1},
$$

with certain coefficients $\bar{x}_{i}$ and a generic constant $C_{\alpha}$ depending on $\alpha$.

In the next theorem, we state and justify the existence and uniqueness theorem for (1.1) along with a smoothness result.

THEOREM 2.2. Let $\alpha=\frac{p}{q} \in(0,1)$ with two relatively prime integers $p \geq 1$ and $q \geq 2$, and assume that the following conditions are satisfied:

(a) $p_{i}(t), q_{j}(t) \in C(I)$, for $i=1, \ldots, 4, j=1,2$,

(b) $p_{4}(t) \neq 0$ on $I$.

Then the fractional-order differential algebraic equation (1.1) has unique continuous solutions $x(t)$ and $y(t)$. Furthermore, if $p_{i}, q_{j}$ are of the form

$$
p_{i}(t)=\bar{p}_{i}\left(t^{\frac{1}{q}}\right), \quad q_{j}(t)=\bar{q}_{j}\left(t^{\frac{1}{q}}\right)
$$

where $\bar{p}_{i}\left(t^{\frac{1}{q}}\right)$ and $\bar{q}_{j}\left(t^{\frac{1}{q}}\right)$ are analytic functions in a neighborhood of the origin, then the following regularity result for the unique solutions of (1.1) hold:

$$
\begin{aligned}
\left|x^{\prime}(t)\right| & \leq C_{\alpha} t^{\alpha-1}, \\
\left|y^{(\tilde{v}+1)}(t)\right| & \leq \tilde{C}_{\alpha} t^{\alpha-1},
\end{aligned}
$$

where $C_{\alpha}, \tilde{C}_{\alpha}$ are generic constants and $\tilde{v} \geq 0$ depends on the regularity of the input functions.

Proof. According to assumption (b), the algebraic constraint of (1.1) can be written as

$$
y(t)=-\frac{1}{p_{4}(t)}\left(p_{3}(t) x(t)+q_{2}(t)\right) .
$$

Replacing (2.4) in (1.1) gives

$$
\begin{aligned}
D^{\alpha} x(t) & =p_{1}(t) x(t)-\frac{p_{2}(t)}{p_{4}(t)}\left(p_{3}(t) x(t)+q_{2}(t)\right)+q_{1}(t) \\
& =\left(\frac{p_{1}(t) p_{4}(t)-p_{2}(t) p_{3}(t)}{p_{4}(t)}\right) x(t)+\left(\frac{q_{1}(t) p_{4}(t)-q_{2}(t) p_{2}(t)}{p_{4}(t)}\right) \\
& =: f(t, x(t)) .
\end{aligned}
$$


Trivially, the linear and continuous function $f(t, x(t))$ defined in (2.5) satisfies the Lipschitz condition with respect to its second variable, and it can be written as $f(t, x(t))=\bar{f}\left(t^{\frac{1}{q}}, x(t)\right)$, where $\bar{f}$ is an analytic function in a neighborhood of $\left(0, d_{0}\right)$ in view of $(2.1)$. Thus, Theorem 2.1 implies a unique continuous solution $x(t)$ with the regularity property (2.2) for the function in (2.5). Furthermore, relation (2.4) indicates that $y(t)$ has at least the same smoothness degree as $x(t)$ depending on the regularity properties of the input functions, which verifies the bound (2.3).

Indeed, from Theorems 2.1 and 2.2 we conclude that the exact solutions $x(t)$ and $y(t)$ of (1.1) are represented by

$$
\begin{aligned}
& x(t)=d_{0}+\sum_{i=p}^{\infty} \tilde{x}_{i} t^{\frac{i}{q}}=d_{0}+\tilde{x}_{p} t^{\alpha}+\ldots \notin C^{1}(I), \\
& y(t)=-\frac{1}{p_{4}(t)}\left(p_{3}(t)\left[d_{0}+\tilde{x}_{p} t^{\alpha}+\ldots\right]+q_{2}(t)\right) \notin C^{\tilde{v}+1}(I), \quad \tilde{v} \geq 0,
\end{aligned}
$$

where $C^{m}(I), m \geq 0$, is the space of all $m$-times continuously differentiable functions on $I$. This implies that some derivatives of the exact solutions of (1.1) have a singularity near the end point $t=0^{+}$with the asymptotic behavior (2.2), (2.3), and thereby a direct application of the Legendre collocation method for the numerical solution of (1.1) may lead to results with low accuracy.

In this paper, we propose a strategy to overcome this difficulty and provide more accurate approximate solutions for (1.1). To this end, we first implement the Legendre collocation method to solve (1.1) numerically and investigate its convergence properties. Here we prove that a direct implementation of this approach leads to approximate solutions with low accuracy. To overcome this weakness, we pursue a regularization strategy using (2.6) so that the resulting equation has a higher degree of regularity. We justify that this regularization process leads to a substantial improvement in the accuracy of the obtained approximate solutions.

3. The Legendre collocation approach. In this section, we investigate the numerical performance of the Legendre collocation method for approximating the solutions of (1.1).

3.1. Numerical approach. Assume that the approximate solutions of (1.1) are given by

$$
\begin{aligned}
& x_{N}(t)=\sum_{i=0}^{N} x_{i} L_{i}(t)=X \bar{L}=X L V_{t}, \\
& y_{N}(t)=\sum_{i=0}^{N} y_{i} L_{i}(t)=Y \bar{L}=Y L V_{t},
\end{aligned}
$$

where $L_{i}(t), i=0,1, \ldots, N$, are the shifted Legendre polynomials on $I$ and

$$
\bar{L}=\left[L_{0}(t), L_{1}(t), \ldots, L_{N}(t)\right]^{T}=L V_{t},
$$

where $L$ is a nonsingular lower triangular coefficient matrix given by

$$
L:=\left[\begin{array}{cccccc}
1 & & & & & \\
-1 & 2 & & & & \\
1 & -6 & 6 & & & \\
-1 & 12 & -30 & 20 & & \\
1 & -20 & 90 & -140 & 70 & \\
\vdots & \vdots & \vdots & \vdots & \vdots & \ddots
\end{array}\right]
$$


and where $V_{t}=\left[1, t, t^{2}, \ldots, t^{N}\right]^{T}$ is the standard basis and $X=\left[x_{0}, x_{1}, \ldots, x_{N}\right]$, $Y=\left[y_{0}, y_{1}, \ldots, y_{N}\right]$ are unknown vectors.

Substituting (3.1), (3.2) into (1.1) yields

$$
\begin{aligned}
X L D^{\alpha} V_{t} & =X L V_{t}^{p_{1}}+Y L V_{t}^{p_{2}}+q_{1}(t), \\
0 & =X L V_{t}^{p_{3}}+Y L V_{t}^{p_{4}}+q_{2}(t),
\end{aligned}
$$

where $V_{t}^{p_{i}}=p_{i}(t) V_{t}, i=1, \ldots, 4$. The second relation of (1.2) gives

$$
\begin{aligned}
D^{\alpha} V_{t} & =\left[D^{\alpha}(1), D^{\alpha}(t), \ldots, D^{\alpha}\left(t^{N}\right)\right]^{T} \\
& =\left[0, \frac{1}{\Gamma(2-\alpha)} t^{1-\alpha}, \ldots, \frac{N !}{\Gamma(N-\alpha+1)} t^{N-\alpha}\right]^{T} \\
& =t^{-\alpha}\left[0, \frac{1}{\Gamma(2-\alpha)} t, \ldots, \frac{N !}{\Gamma(N-\alpha+1)} t^{N}\right]^{T}=\Omega_{t} V_{t},
\end{aligned}
$$

where $\Omega_{t}$ is the following $(N+1) \times(N+1)$ diagonal matrix

$$
\Omega_{t}=\left[\begin{array}{ccccc}
0 & 0 & 0 & \cdots & 0 \\
0 & \frac{t^{-\alpha}}{\Gamma(2-\alpha)} & 0 & \cdots & 0 \\
0 & 0 & \frac{2 t^{-\alpha}}{\Gamma(3-\alpha)} & \cdots & 0 \\
\vdots & \vdots & \vdots & \ddots & 0 \\
0 & 0 & 0 & \cdots & \frac{t^{-\alpha} N !}{\Gamma(N+1-\alpha)}
\end{array}\right]
$$

Inserting (3.4) into (3.3) yields

$$
\begin{aligned}
X L \Omega_{t} V_{t} & =X L V_{t}^{p_{1}}+Y L V_{t}^{p_{2}}+q_{1}(t), \\
0 & =X L V_{t}^{p_{3}}+Y L V_{t}^{p_{4}}+q_{2}(t) .
\end{aligned}
$$

In the collocation method, the unknown vectors $X$ and $Y$ are obtained by imposing the condition that equation (3.5) is satisfied at a set of suitable nodal points. Here we choose the Legendre-Gauss nodal points $\left\{t_{j}\right\}_{j=0}^{N}$ as collocation points, which are the zeros of the polynomial $L_{N+1}(t)$ [15]. Inserting the collocation points into (3.5), the following $(2 N+2) \times(2 N+2)$ system of linear algebraic equations for the unknown vectors $X$ and $Y$ is obtained:

$$
\begin{aligned}
X L \Omega_{t_{j}} V_{t_{j}} & =X L V_{t_{j}}^{p_{1}}+Y L V_{t_{j}}^{p_{2}}+q_{1}\left(t_{j}\right), \quad j=0,1, \ldots, N . \\
0 & =X L V_{t_{j}}^{p_{3}}+Y L V_{t_{j}}^{p_{4}}+q_{2}\left(t_{j}\right), \quad
\end{aligned}
$$

Finally, the unknown vectors $X, Y$ and thereby the approximate solutions $x_{N}(t), y_{N}(t)$ defined by (3.1) and (3.2) can be defined by requiring the initial conditions

$$
\begin{aligned}
& x_{N}(0)=X\left[L_{i}(0)\right]_{i=0}^{N}=X\left[(-1)^{i}\right]_{i=0}^{N}=d_{0}, \\
& y_{N}(0)=Y\left[L_{i}(0)\right]_{i=0}^{N}=Y\left[(-1)^{i}\right]_{i=0}^{N}=d_{1},
\end{aligned}
$$

additionally to the linear system (3.6).

3.2. Convergence analysis. In this section, we investigate the convergence properties of the proposed Legendre collocation method for the numerical solution of (1.1). For simplicity we consider (1.1) when its coefficients $p_{i}, i=1, \ldots, 4$, are constants. Throughout this paper, $C$ and $C_{i}$ are generic positive constants independent of $N$. In the following, we recall some useful preliminaries and lemmas which will be used in the sequel. 
- $L_{\delta, \gamma}^{2}$ is the space of all functions $u$ for which $\|u\|_{\delta, \gamma}<\infty$ with

$$
\|u\|_{\delta, \gamma}^{2}=\int_{I}|u(t)|^{2} \omega^{\delta, \gamma}(t) d t,
$$

where $\omega^{\delta, \gamma}(t)=2^{\delta+\gamma} t^{\gamma}(1-t)^{\delta}$ is the shifted Jacobi weight function on $I$ for the parameters $\delta, \gamma>-1$. For simplicity, we use the symbol $\left(L^{2}(I),\|\cdot\|\right)$ when $\delta=\gamma=0$ (see [15]).

- $B^{m}(I)$ is the non-uniform Sobolev space of all function $u(t)$ on $I$ with $\|u\|_{m}<\infty$, where

$$
\|u\|_{m}^{2}=\sum_{k=0}^{m}\left\|u^{(k)}\right\|_{k, k}^{2} .
$$

The semi-norm $|u|_{m}=\left\|u^{(m)}\right\|_{m, m}$ can also be defined in this space (see [15]).

- $\omega(u ; \nu)$ is the modulus of continuity of the function $u(t)$ on $I$ defined for $x_{1}, x_{2} \in I$ and $\nu>0$ by (see [13])

$$
\omega(u ; \nu)=\sup _{\left|x_{1}-x_{2}\right| \leq \nu}\left|u\left(x_{1}\right)-u\left(x_{2}\right)\right| .
$$

We need the following property of the modulus of continuity:

LEMMA 3.1 ([13]). $u(t)$ is uniformly continuous on I if and only if

$$
\lim _{\nu \rightarrow 0} \omega(u ; \nu)=0 .
$$

- The Lagrange interpolating polynomial $I_{N}(u(t))$ for any continuous function $u(t)$ on $I$ is defined by

$$
I_{N}(u(t))=\sum_{i=0}^{N} u\left(t_{i}\right) \varphi_{i}(t),
$$

where $\left\{\varphi_{i}(t)\right\}_{i=0}^{N}$ are the Lagrange polynomials associated with the Legendre-Gauss points $\left\{t_{i}\right\}_{i=0}^{N}$.

In our analysis we shall apply the following lemmas:

LEMMA 3.2 (Theorem 3.41 and Remark 4.14 in [15]). For any $u \in B^{m}(I)$ with a fixed $m \geq 1$, we have

$$
\left\|u-I_{N}(u)\right\| \leq C N^{-m}|u|_{m} .
$$

LEMMA 3.3 (Corollary 1.4.1 and Theorem 4.8 in [13]). For any continuous function $u(t)$ on I we have

$$
\left\|u-I_{N}(u)\right\| \leq L \omega\left(u ; \frac{1}{2 N}\right),
$$

where $L$ is a known constant independent of $N$.

Lemma 3.4 ([12, Lemma 2.1(a)]). The Riemann-Liouville-type fractional integral operator $I^{\alpha}$ is bounded on $L^{2}(I)$, i.e.,

$$
\left\|I^{\alpha} u\right\| \leq C\|u\|, \quad u \in L^{2}(I) .
$$


LEMMA 3.5 ([10] (Gronwall's inequality)). Let the non-negative and locally integrable function $u(t)$ satisfy the inequality

$$
u(t) \leq b(t)+d \int_{0}^{t}(t-s)^{m} u(s) d s, \quad s \in I, \quad m>-1, \quad d \geq 0, \quad b(t) \geq 0 .
$$

Then there exists a constant $C$ such that

$$
u(t) \leq b(t)+C \int_{0}^{t}(t-s)^{m} b(s) d s, \quad s \in I .
$$

The next theorem gives suitable bounds for the error functions of the Legendre collocation approximations for (1.1) with constant coefficients.

THEOREM 3.6. Let the requirements of Theorem 2.2 hold. Moreover, assume that $x_{N}(t)$, $y_{N}(t)$ are the Legendre collocation approximations (3.1) and (3.2) to the exact solutions $x(t)$ and $y(t)$ of the fractional differential algebraic equation (1.1) with constant coefficients. If $q_{i}(t) \in B^{k_{i}}(I)$, for $i=1,2$, then for sufficiently large $N$ we have

$$
\begin{aligned}
\left\|e_{N}\right\| & \leq C\left(N^{-k_{1}}\left|q_{1}\right|_{k_{1}}+\left|\frac{p_{2}}{p_{4}}\right| N^{-k_{2}}\left|q_{2}\right|_{k_{2}}+N^{-1}\left|D^{\alpha} x\right|_{1}\right), \\
\left\|\varepsilon_{N}\right\| & \leq C\left(\left|\frac{p_{3}}{p_{4}}\right|\left\|e_{N}\right\|+N^{-k_{2}}\left|q_{2}\right|_{k_{2}}\right),
\end{aligned}
$$

where $e_{N}(t)=x(t)-x_{N}(t)$ and $\varepsilon_{N}(t)=y(t)-y_{N}(t)$.

Proof. Considering (1.1) with constant coefficients, by the described strategy in the previous section, we obtain

$$
\begin{aligned}
I_{N}\left(D^{\alpha} x_{N}(t)\right) & =p_{1} x_{N}(t)+p_{2} y_{N}(t)+I_{N}\left(q_{1}\right), \\
0 & =p_{3} x_{N}(t)+p_{4} y_{N}(t)+I_{N}\left(q_{2}\right) .
\end{aligned}
$$

Subtracting (1.1) from (3.8) and some simple computations give

$$
\begin{aligned}
D^{\alpha} e_{N}(t) & =p_{1} e_{N}(t)+p_{2} \varepsilon_{N}(t)-e_{I_{N}}\left(D^{\alpha} x_{N}(t)\right)+e_{I_{N}}\left(q_{1}\right), \\
0 & =p_{3} e_{N}(t)+p_{4} \varepsilon_{N}(t)+e_{I_{N}}\left(q_{2}\right),
\end{aligned}
$$

where $e_{I_{N}}(u)=u-I_{N}(u)$ is the interpolating error function. Since $p_{4} \neq 0$, the algebraic constraint in (3.9) has the form

$$
\varepsilon_{N}(t)=-\frac{1}{p_{4}}\left(e_{I_{N}}\left(q_{2}\right)+p_{3} e_{N}(t)\right) .
$$

Replacing (3.10) in (3.9) yields

$$
D^{\alpha} e_{N}(t)=\left(p_{1}-\frac{p_{3} p_{2}}{p_{4}}\right) e_{N}(t)-\frac{p_{2}}{p_{4}} e_{I_{N}}\left(q_{2}\right)+e_{I_{N}}\left(q_{1}\right)-e_{I_{N}}\left(D^{\alpha} x_{N}\right) .
$$

By applying the fractional integral operator $I^{\alpha}$ on both sides of (3.11) and using the first relation in (1.2), we conclude that

$$
e_{N}(t)=\left(p_{1}-\frac{p_{3} p_{2}}{p_{4}}\right) I^{\alpha} e_{N}(t)-\frac{p_{2}}{p_{4}} I^{\alpha}\left(e_{I_{N}}\left(q_{2}\right)\right)+I^{\alpha}\left(e_{I_{N}}\left(q_{1}\right)\right)-I^{\alpha}\left(e_{I_{N}}\left(D^{\alpha} x_{N}\right)\right),
$$


which can be written as

$$
\left|e_{N}\right| \leq d \int_{0}^{t}(t-s)^{\alpha-1}\left|e_{N}(s)\right| d s+b(t)
$$

where

$$
\begin{aligned}
b(t) & =\left|\frac{p_{2}}{p_{4}}\right| I^{\alpha}\left(\left|e_{I_{N}}\left(q_{2}\right)\right|\right)+I^{\alpha}\left(\left|e_{I_{N}}\left(q_{1}\right)\right|\right)+I^{\alpha}\left(\left|e_{I_{N}}\left(D^{\alpha} x_{N}\right)\right|\right), \\
d & =\left|\left(p_{1}-\frac{p_{3} p_{2}}{p_{4}}\right) \frac{1}{\Gamma(\alpha)}\right| .
\end{aligned}
$$

By applying Gronwall's inequality, i.e., Lemma 3.5, to (3.12), we have

and thereby

$$
\left|e_{N}\right| \leq b(t)+C \int_{0}^{t}(t-s)^{\alpha-1} b(s) d s=b(t)+C \Gamma(\alpha) I^{\alpha}(b(t)),
$$

$$
\left\|e_{N}\right\| \leq\|b(t)\|+|C \Gamma(\alpha)|\left\|I^{\alpha}(b(t))\right\|,
$$

where $C$ is a constant. Using the boundedness of $I^{\alpha}$, i.e., Lemma 3.4, in the inequality above, we obtain

$$
\begin{aligned}
\left\|e_{N}\right\| & \leq C_{1}\left(\left|\frac{p_{2}}{p_{4}}\right|\left\|I^{\alpha}\left(e_{I_{N}}\left(q_{2}\right)\right)\right\|+\left\|I^{\alpha}\left(e_{I_{N}}\left(q_{1}\right)\right)\right\|+\left\|I^{\alpha}\left(e_{I_{N}}\left(D^{\alpha} x_{N}\right)\right)\right\|\right) \\
\left\|e_{N}\right\| & \leq C_{2}\left(\left|\frac{p_{2}}{p_{4}}\right|\left\|e_{I_{N}}\left(q_{2}\right)\right\|+\left\|e_{I_{N}}\left(q_{1}\right)\right\|+\left\|e_{I_{N}}\left(D^{\alpha} x_{N}\right)\right\|\right) \\
& \leq C_{2}\left(\left|\frac{p_{2}}{p_{4}}\right|\left\|e_{I_{N}}\left(q_{2}\right)\right\|+\left\|e_{I_{N}}\left(q_{1}\right)\right\|+\left\|e_{I_{N}}\left(D^{\alpha} x\right)\right\|+\left\|e_{I_{N}}\left(D^{\alpha} e_{N}\right)\right\|\right) .
\end{aligned}
$$

Now, we find suitable bounds for each term on the right-hand side of (3.13). To this end, using (3.1) and (1.2), we may write

$$
D^{\alpha} x_{N}(t)=D^{\alpha}\left(\sum_{i=0}^{N} x_{i} L_{i}(t)\right)=D^{\alpha}\left(\sum_{i=0}^{N} \hat{x}_{i} t^{i}\right)=\sum_{i=0}^{N} \hat{x}_{i} D^{\alpha} t^{i}=\sum_{i=1}^{N} \frac{i ! \hat{x}_{i}}{\Gamma(i-\alpha+1)} t^{i-\alpha},
$$

which implies continuity of $D^{\alpha} x_{N}$ for $0<\alpha<1$. On the other hand, from relation (2.6), we have

$$
\begin{aligned}
D^{\alpha} x(t) & =D^{\alpha}\left(d_{0}+\sum_{i=p}^{\infty} \bar{x}_{i} t^{\frac{i}{q}}\right)=\sum_{i=p}^{\infty} \bar{x}_{i} D^{\alpha}\left(t^{\frac{i}{q}}\right)=\sum_{i=p}^{\infty} \frac{\Gamma\left(\frac{i}{q}+1\right) \bar{x}_{i}}{\Gamma\left(\frac{i-p}{q}+1\right)} t^{\frac{i-p}{q}} \\
& =\Gamma(\alpha+1) \bar{x}_{p}+\frac{\Gamma\left(\alpha+\frac{1}{q}+1\right) \bar{x}_{p+1}}{\Gamma\left(\frac{1}{q}+1\right)} t^{\frac{1}{q}}+\ldots,
\end{aligned}
$$

which indicates that $D^{\alpha} x(t)$ and consequently $D^{\alpha} e_{N}(t)=D^{\alpha} x(t)-D^{\alpha} x_{N}(t)$ are continuous functions on $I$. Therefore using Lemma 3.3 we obtain

$$
\left\|e_{I_{N}}\left(D^{\alpha} e_{N}\right)\right\| \leq L \omega\left(D^{\alpha} e_{N} ; \frac{1}{2 N}\right) .
$$


Substituting (3.15) into (3.13) yields

$$
\left\|e_{N}\right\| \leq C_{3}\left(\left|\frac{p_{2}}{p_{4}}\right|\left\|e_{I_{N}}\left(q_{2}\right)\right\|+\left\|e_{I_{N}}\left(q_{1}\right)\right\|+\left\|e_{I_{N}}\left(D^{\alpha} x\right)\right\|+\omega\left(D^{\alpha} e_{N} ; \frac{1}{2 N}\right)\right) .
$$

Since any continuous function on a compact set is uniformly continuous (by the Heine-Cantor theorem [14]), $D^{\alpha} e_{N}(t)$ is a uniformly continuous function on the closed interval $I$. Then Lemma 3.1 yields

$$
\omega\left(D^{\alpha} e_{N} ; \frac{1}{2 N}\right) \rightarrow 0 \quad \text { as } N \rightarrow \infty,
$$

and equivalently, for any $\epsilon>0$, there exists a positive number $N_{\epsilon}$ such that for $N \geq N_{\epsilon}$, we have

$$
\left|\omega\left(D^{\alpha} e_{N} ; \frac{1}{2 N}\right)\right| \leq \epsilon .
$$

Thus, for sufficiently small values of $\epsilon$ we can ignore $\omega\left(D^{\alpha} e_{N}, \frac{1}{2 N}\right)$ in (3.16) for sufficiently large $N$, and so it can be estimated by

$$
\left\|e_{N}\right\| \leq C_{3}\left(\left|\frac{p_{2}}{p_{4}}\right|\left\|e_{I_{N}}\left(q_{2}\right)\right\|+\left\|e_{I_{N}}\left(q_{1}\right)\right\|+\left\|e_{I_{N}}\left(D^{\alpha} x\right)\right\|\right) .
$$

The first inequality in (3.7) can be obtained by applying Lemma 3.2 to (3.17) and using

$$
\left|D^{\alpha} x\right|_{1}=\left\|\frac{d}{d t}\left(D^{\alpha} x\right)\right\|_{1,1}<\infty
$$

in view of (3.14).

Finally, the second inequality in (3.7) can be obtained by applying the $L^{2}$-norm on both sides of (3.10) and using Lemma 3.2.

From Theorem 3.6, we can deduce that a singularity of the exact solutions of (1.1) near $t=0^{+}$yields a loss in the order of convergence of the Legendre collocation method. In the next section, for recovering the high order of convergence, we introduce a regularization approach which enables us to retrieve the regularity of the exact solutions and produce approximate solutions for (1.1) of higher accuracy.

4. Establishing convergence of higher order. In this section, we introduce a regularization approach that enables us to improve the differentiability of the input functions and then to define approximate solutions with a high rate of convergence for (1.1). To this end, under the assumptions of Theorem 2.2, we use the coordinate transformations

$$
t=u^{q}, \quad u=t^{\frac{1}{q}}, \quad s=w^{q}, \quad w=s^{\frac{1}{q}},
$$

and transform equation (1.1) into the following equivalent one:

$$
\left\{\begin{aligned}
\bar{D}^{\alpha} \bar{x}(u) & =\bar{p}_{1}(u) \bar{x}(u)+\bar{p}_{2}(u) \bar{y}(u)+\bar{q}_{1}(u), \\
0 & =\bar{p}_{3}(u) \bar{x}(u)+\bar{p}_{4}(u) \bar{y}(u)+\bar{q}_{2}(u), \\
\bar{x}(0) & =d_{0}, \quad \bar{y}(0)=d_{1}, \quad u \in I,
\end{aligned}\right.
$$

where

$$
\bar{p}_{i}(u)=p_{i}\left(u^{q}\right), \quad i=1,2, \ldots, 4, \quad \bar{q}_{j}(u)=q_{j}\left(u^{q}\right), \quad j=1,2,
$$


are analytic functions and

$$
\bar{D}^{\alpha} \bar{x}(u)=\frac{1}{\Gamma(1-\alpha)} \int_{0}^{u}\left(u^{q}-w^{q}\right)^{-\alpha}(\bar{x}(w))^{\prime} d w .
$$

Following (2.6), the exact solutions $\bar{x}(u)$ and $\bar{y}(u)$ of the transformed equation (4.2) can be represented as

$$
\begin{aligned}
& \bar{x}(u)=x\left(u^{q}\right)=d_{0}+\sum_{i=p}^{\infty} \tilde{x}_{i} u^{i}=d_{0}+\tilde{x}_{p} u^{p}+\tilde{x}_{p+1} u^{p+1} \ldots, \\
& \bar{y}(u)=y\left(u^{q}\right)=-\frac{1}{\bar{p}_{4}(u)}\left(\bar{p}_{3}(u)\left[d_{0}+\sum_{i=p}^{\infty} \tilde{x}_{i} u^{i}\right]+\bar{q}_{2}(u)\right),
\end{aligned}
$$

which are infinitely smooth functions. Thus, the smoothing transformation (4.1) removes the singularity of $x(t)$ and $y(t)$ in (2.6). This enables us to provide highly accurate approximate solutions for (1.1) by solving the equivalent equation (4.2) with the Legendre collocation method.

4.1. Numerical approach. Let the Legendre collocation solutions of (4.2) be given by

$$
\begin{aligned}
& \bar{x}_{N}(u)=\sum_{i=0}^{N} \bar{x}_{i} L_{i}(u)=\bar{X} \bar{L}=\bar{X} L V_{u}, \\
& \bar{y}_{N}(u)=\sum_{i=0}^{N} \bar{y}_{i} L_{i}(u)=\bar{Y} \bar{L}=\bar{Y} L V_{u},
\end{aligned}
$$

where $\bar{X}=\left[\bar{x}_{0}, \bar{x}_{1}, \ldots, \bar{x}_{N}\right], \bar{Y}=\left[\bar{y}_{0}, \bar{y}_{1}, \ldots, \bar{y}_{N}\right]$ are unknown vectors. Since the unknown solutions of (1.1) can be written as $x(t)=\bar{x}(u), y(t)=\bar{y}(u)$, we consider

$$
\widetilde{x}_{N}(t)=\bar{x}_{N}\left(t^{\frac{1}{q}}\right)=\bar{x}_{N}(u), \quad \widetilde{y}_{N}(t)=\bar{y}_{N}\left(t^{\frac{1}{q}}\right)=\bar{y}_{N}(u),
$$

as approximate solutions for the main equation (1.1).

Inserting (4.3) and (4.4) into (4.2) we find

$$
\begin{aligned}
\bar{X} L \bar{D}^{\alpha} V_{u} & =\bar{X} L V_{u}^{\bar{p}_{1}}+\bar{Y} L V_{u}^{\bar{p}_{2}}+\bar{q}_{1}(u), \\
0 & =\bar{X} L V_{u}^{\bar{p}_{3}}+\bar{Y} L V_{u}^{\bar{p}_{4}}+\bar{q}_{2}(u),
\end{aligned}
$$

where $V_{u}^{\bar{p}_{i}}=\bar{p}_{i}(u) V_{u}, i=1, \ldots, 4$. To obtain the matrix representation of $\bar{D}^{\alpha} V_{u}$ we can write

$$
\bar{D}^{\alpha} V_{u}=\frac{1}{\Gamma(1-\alpha)} \int_{0}^{u}\left(u^{q}-w^{q}\right)^{-\alpha}\left(V_{w}\right)^{\prime} d w .
$$

From $\left(V_{w}\right)^{\prime}=\eta V_{w}$ (see [11]), where $\eta$ is the $(N+1) \times(N+1)$ matrix

$$
\eta=\left[\begin{array}{ccccc}
0 & 0 & 0 & \ldots & 0 \\
1 & 0 & 0 & \ldots & 0 \\
0 & 2 & 0 & \ldots & 0 \\
0 & 0 & 3 & \ldots & 0 \\
\vdots & \vdots & \vdots & \ddots & \vdots \\
0 & 0 & 0 & \ldots & N
\end{array}\right]
$$


we get

$$
\bar{D}^{\alpha} V_{u}=\frac{\eta}{\Gamma(1-\alpha)}\left[\int_{0}^{u}\left(u^{q}-w^{q}\right)^{-\alpha} w^{j} d w\right]_{j=0}^{N} .
$$

Using the variable transformation $w^{q}=u^{q} v$, we have

$$
\int_{0}^{u}\left(u^{q}-w^{q}\right)^{-\alpha} w^{j} d w=u^{j-p+1} \frac{B\left(\frac{j+1}{q}, 1-\alpha\right)}{q}, \quad j=0,1, \ldots, N,
$$

where $B(a, b)=\int_{0}^{1} t^{a-1}(1-t)^{b-1} d t$ is the Beta function. Thus, by ignoring the first $p-1$ terms in (4.7), we can write

$$
\bar{D}^{\alpha} V_{u}=\eta \bar{\Omega} V_{u},
$$

where $\bar{\Omega}$ is the $(N+1) \times(N+1)$ matrix with the only nonzero entries

$$
\bar{\Omega}_{j, j-p+1}=\frac{B\left(1-\alpha, \frac{j+1}{q}\right)}{q}, \quad j=p-1, p, \ldots, N .
$$

Substituting (4.8) into (4.6) yields

$$
\begin{aligned}
\bar{X} L \eta \bar{\Omega} V_{u} & =\bar{X} L V_{u}^{\bar{p}_{1}}+\bar{Y} L V_{u}^{\bar{p}_{2}}+\bar{q}_{1}(u), \\
0 & =\bar{X} L V_{u}^{\bar{p}_{3}}+\bar{Y} L V_{u}^{\bar{p}_{4}}+\bar{q}_{2}(u) .
\end{aligned}
$$

Inserting the Legendre-Gauss points $\left\{t_{j}\right\}_{j=0}^{N}$ into equations (4.9) yields the following $(2 N+2) \times(2 N+2)$ system of linear algebraic equations for the unknown vectors $\bar{X}$ and $\bar{Y}$ :

$$
\begin{aligned}
\bar{X} L \eta \bar{\Omega} V_{t_{j}} & =\bar{X} L V_{t_{j}}^{\bar{p}_{1}}+\bar{Y} L V_{t_{j}}^{\bar{p}_{2}}+\bar{q}_{1}\left(t_{j}\right), \quad j=0,1, \ldots, N . \\
0 & =\bar{X} L V_{t_{j}}^{\bar{p}_{3}}+\bar{Y} L V_{t_{j}}^{\bar{p}_{4}}+\bar{q}_{2}\left(t_{j}\right), \quad
\end{aligned}
$$

Finally, the unknown vectors $\bar{X}$ and $\bar{Y}$ and thereby the approximate solutions $\bar{x}_{N}(t), \bar{y}_{N}(t)$ defined by (4.3) and (4.4) can be obtained by imposing the initial conditions

$$
\begin{aligned}
& \bar{x}_{N}(0)=\bar{X}\left[L_{i}(0)\right]_{i=0}^{N}=\bar{X}\left[(-1)^{i}\right]_{i=0}^{N}=d_{0}, \\
& \bar{y}_{N}(0)=\bar{Y}\left[L_{i}(0)\right]_{i=0}^{N}=\bar{Y}\left[(-1)^{i}\right]_{i=0}^{N}=d_{1},
\end{aligned}
$$

additionally to the linear system (4.10). The proposed scheme is summarized in Algorithm 1.

4.2. Convergence analysis. The main objective of this section is to investigate the effect of the proposed regularization procedure for increasing the rate of convergence of the approximate solutions (4.5).

THEOREM 4.1. Let $\bar{x}_{N}(u), \bar{y}_{N}(u)$, defined by (4.3) and (4.4), respectively, be the Legendre collocation approximations of (4.2) with constant coefficients. Moreover, assume that $\widetilde{x}_{N}(t), \widetilde{y}_{N}(t)$ given by (4.5) are the approximate solutions to the fractional differential algebraic equation (1.1) with constant coefficients. If $\bar{q}_{i}(u) \in B^{k_{i}}(I)$, for $i=1,2$ and $k_{i}>0$, then for sufficiently large $N$, we obtain

$$
\begin{aligned}
& \left\|\widetilde{e}_{N}\right\|_{0, q-1} \leq C\left(N^{-k_{1}}\left|\bar{q}_{1}\right|_{k_{1}}+\left|\frac{p_{2}}{p_{4}}\right| N^{-k_{2}}\left|\bar{q}_{2}\right|_{k_{2}}\right), \\
& \left\|\widetilde{\varepsilon}_{N}\right\|_{0, q-1} \leq C\left(\left|\frac{p_{3}}{p_{4}}\right|\left\|\widetilde{e}_{N}\right\|_{0, q-1}+N^{-k_{2}}\left|\bar{q}_{2}\right|_{k_{2}}\right),
\end{aligned}
$$

where $\widetilde{e}_{N}(t)=x(t)-\widetilde{x}_{N}(t)$ and $\widetilde{\varepsilon}_{N}(t)=y(t)-\widetilde{y}_{N}(t)$ are the error functions. 
Algorithm 1 The construction of Legendre collocation approximation system (4.10).

Step 1. Choose $N$ and compute the coefficient matrix $L$ from $\left\{L_{i}(u)\right\}_{i=0}^{N}=L V_{u}$ with $\mathcal{O}\left(n^{2}\right)$ flops and $V_{u}=\left[1, u, \ldots, u^{N}\right]^{T}$.

Step 2. Compute the $(N+1) \times(N+1)$ matrices $\eta$ and $\bar{\Omega}$ with the only nonzero entries

$$
\begin{aligned}
& \eta_{i+1, i}=i, \quad i=0,1, \ldots, N, \\
& \bar{\Omega}_{j, j-p+1}=\frac{B\left(1-\alpha, \frac{j+1}{q}\right)}{q}, \quad j=p-1, \ldots, N,
\end{aligned}
$$

with $\mathcal{O}(N)$ flops.

Step 3. Set the Legendre-Gauss points $\left\{t_{j}\right\}_{j=0}^{N}$ as the zeros of the Legendre polynomials $L_{N+1}(t)$.

Step 4. Compute the unknown vectors $\bar{X}$ and $\bar{Y}$ from solving the $(2 N+2) \times(2 N+2)$ linear system of equations (4.10) after imposing the initial conditions and set $\bar{x}_{N}(u)=\bar{X} L V_{u}, \bar{y}_{N}(u)=\bar{Y} L V_{u}$, as the Legendre collocation solutions of the transformed equation (4.2).

Step 5. Set $\widetilde{x}_{N}(t)=\bar{x}_{N}\left(t^{\frac{1}{q}}\right), \widetilde{y}_{N}(t)=\bar{y}_{N}\left(t^{\frac{1}{q}}\right)$ as the approximate solutions of the main equation (1.1).

Proof. From (4.8) it can be seen that $\bar{D}^{\alpha}\left(\bar{x}_{N}(u)\right)$ is a polynomial of degree at most $N$. Thus, we can write $I_{N}\left(\bar{D}^{\alpha}\left(\bar{x}_{N}(u)\right)\right)=\bar{D}^{\alpha}\left(\bar{x}_{N}(u)\right)$, and thereby, applying the Legendre collocation scheme to the equation (4.2) with constant coefficients yields

$$
\begin{aligned}
\bar{D}^{\alpha} \bar{x}_{N}(u) & =p_{1} \bar{x}_{N}(u)+p_{2} \bar{y}_{N}(u)+I_{N}\left(\bar{q}_{1}(u)\right), \\
0 & =p_{3} \bar{x}_{N}(u)+p_{4} \bar{y}_{N}(u)+I_{N}\left(\bar{q}_{2}(u)\right) .
\end{aligned}
$$

Subtracting (4.2) from (4.12), we find

$$
\begin{aligned}
\bar{D}^{\alpha} \bar{e}_{N}(u) & =p_{1} \bar{e}_{N}(u)+p_{2} \bar{\varepsilon}_{N}(u)+e_{I_{N}}\left(\bar{q}_{1}(u)\right), \\
0 & =p_{3} \bar{e}_{N}(u)+p_{4} \bar{\varepsilon}_{N}(u)+e_{I_{N}}\left(\bar{q}_{2}(u)\right) .
\end{aligned}
$$

Since $p_{4} \neq 0$, the algebraic constraint of (4.13) has the form

$$
\bar{\varepsilon}_{N}(u)=-\frac{1}{p_{4}}\left(p_{3} \bar{e}_{N}(u)+e_{I_{N}}\left(\bar{q}_{2}\right)\right) .
$$

Replacing (4.14) in (4.13), we have

$$
\bar{D}^{\alpha} \bar{e}_{N}(u)=\left(p_{1}-\frac{p_{2} p_{3}}{p_{4}}\right) \bar{e}_{N}(u)-\frac{p_{2}}{p_{4}} e_{I_{N}}\left(\bar{q}_{2}\right)+e_{I_{N}}\left(\bar{q}_{1}\right) .
$$

Applying the transformation (4.1) to the fractional integral operator $I^{\alpha}$, we get

$$
\bar{I}^{\alpha}(\bar{x})=\frac{1}{\Gamma(\alpha)} \int_{0}^{u}\left(u^{q}-w^{q}\right)^{\alpha-1} \bar{x}(w) q w^{q-1} d w .
$$

From the first relation of (1.2) it is easy to see that

$$
\bar{I}^{\alpha}\left(\bar{D}^{\alpha}\left(\bar{e}_{N}(u)\right)\right)=\bar{e}_{N}(u)-\bar{e}_{N}(0)=\bar{e}_{N}(u) .
$$


Applying the operator $\bar{I}^{\alpha}$ on both sides of (4.15) and using (4.16), we can conclude that

$$
\bar{e}_{N}(u)=\left(p_{1}-\frac{p_{2} p_{3}}{p_{4}}\right) \bar{I}^{\alpha}\left(\bar{e}_{N}(u)\right)-\frac{p_{2}}{p_{4}} \bar{I}^{\alpha}\left(e_{I_{N}}\left(\bar{q}_{2}\right)\right)+\bar{I}^{\alpha}\left(\left(e_{I_{N}}\left(\bar{q}_{1}\right)\right) .\right.
$$

From (4.1) and (4.5) we can write

$$
\bar{e}_{N}(u)=\bar{x}(u)-\bar{x}_{N}(u)=x\left(u^{q}\right)-\widetilde{x}_{N}(t)=x(t)-\widetilde{x}_{N}(t)=\widetilde{e}_{N}(t),
$$

which implies that (4.17) can be expressed as

$$
\widetilde{e}_{N}(t)=\left(p_{1}-\frac{p_{2} p_{3}}{p_{4}}\right) \bar{I}^{\alpha}\left(\bar{e}_{N}(u)\right)-\frac{p_{2}}{p_{4}} \bar{I}^{\alpha}\left(e_{I_{N}}\left(\bar{q}_{2}\right)\right)+\bar{I}^{\alpha}\left(\left(e_{I_{N}}\left(\bar{q}_{1}\right)\right) .\right.
$$

Applying the variable transformation (4.1) in $\bar{I}^{\alpha}\left(\bar{e}_{N}(u)\right)$ and using (4.18), it is easy to see that $\bar{I}^{\alpha}\left(\bar{e}_{N}(u)\right)=I^{\alpha}\left(\widetilde{e}_{N}(t)\right)$, and then equation (4.19) can be written as

$$
\left|\widetilde{e}_{N}(t)\right| \leq\left|p_{1}-\frac{p_{2} p_{3}}{p_{4}}\right| I^{\alpha}\left(\left|\widetilde{e}_{N}(t)\right|\right)+\left|\frac{p_{2}}{p_{4}}\right| \bar{I}^{\alpha}\left(\left|e_{I_{N}}\left(\bar{q}_{2}\right)\right|\right)+\bar{I}^{\alpha}\left(\left|e_{I_{N}}\left(\bar{q}_{1}\right)\right|\right) .
$$

Proceeding in the same manner for (3.12)-(3.13), inequality (4.20) can be expressed as

$$
\left\|\widetilde{e}_{N}\right\|_{0, q-1} \leq C_{1}\left(\left|\frac{p_{2}}{p_{4}}\right|\left\|\bar{I}^{\alpha}\left(e_{I_{N}}\left(\bar{q}_{2}\right)\right)\right\|_{0, q-1}+\left\|\bar{I}^{\alpha}\left(e_{I_{N}}\left(\bar{q}_{1}\right)\right)\right\|_{0, q-1}\right) .
$$

Using the Cauchy-Schwarz inequality and by some simple computations, we obtain

$$
\begin{aligned}
& \left\|\bar{I}^{\alpha}(v)\right\|_{0, q-1}^{2}=\int_{0}^{1}\left(\int_{0}^{u} \frac{q w^{q-1}}{\Gamma(\alpha)\left(u^{q}-w^{q}\right)^{1-\alpha}} v(w) d w\right)^{2}(2 u)^{q-1} d u \\
& \leq \int_{0}^{1}\left(\left[\int_{0}^{u} \frac{q w^{q-1}}{\Gamma(\alpha)\left(u^{q}-w^{q}\right)^{1-\alpha}} d w\right]\left[\int_{0}^{u} \frac{q w^{q-1}}{\Gamma(\alpha)\left(u^{q}-w^{q}\right)^{1-\alpha}} v^{2}(w) d w\right]\right)(2 u)^{q-1} d u \\
& \leq \frac{q 2^{q-1}}{\Gamma(\alpha)}\left(\int_{0}^{1} \int_{0}^{u} \frac{q w^{q-1}}{\Gamma(\alpha)\left(u^{q}-w^{q}\right)^{1-\alpha}} v^{2}(w) u^{q-1} d w d u\right)
\end{aligned}
$$

$$
\leq \frac{q 2^{q-1}}{\Gamma^{2}(\alpha)}\left[\int_{0}^{1} v^{2}(w)\left(\int_{w}^{1} \frac{q u^{q-1}}{\left(u^{q}-w^{q}\right)^{1-\alpha}} d u\right) d w\right] \leq \frac{q^{2} 2^{q-1}}{\Gamma^{2}(\alpha)}\|v\| .
$$

Inserting (4.22) into (4.21) yields

$$
\left\|\widetilde{e}_{N}\right\|_{0, q-1} \leq C_{2}\left(\left|\frac{p_{2}}{p_{4}}\right|\left\|e_{I_{N}}\left(\bar{q}_{2}\right)\right\|+\left\|e_{I_{N}}\left(\bar{q}_{1}\right)\right\|\right) .
$$

Obviously, the first estimate in (4.11) can be obtained by using Lemma 3.2 in the estimate (4.23). In addition, the second estimate in (4.11) can be derived using (4.14), (4.18), Lemma 3.2, and by some simple manipulations.

Using Theorem 4.1 and applying the proposed regularization process, we can approximate the fractional differential algebraic equation (1.1) by a highly accurate numerical solution because the rate of convergence depends on the smoothness of the input functions $\bar{q}_{1}(u)$ and $\bar{q}_{2}(u)$, which have better regularity compared to $q_{1}(t)$ and $q_{2}(t)$. 
5. Numerical examples. In this section, we illustrate by some examples the efficiency of the proposed approach for solving (1.1) numerically. All the computations were done by the Mathematica software. In the tables, the numerical errors are calculated using the norms

$$
\begin{array}{rlrl}
\left\|e_{N}\right\|^{2} & =\int_{I}\left|x(t)-x_{N}(t)\right|^{2} d t, & \left\|\widetilde{e}_{N}\right\|^{2}=\int_{I}\left|x(t)-\widetilde{x}_{N}(t)\right|^{2} d t, \\
\left\|\varepsilon_{N}\right\|^{2}=\int_{I}\left|y(t)-y_{N}(t)\right|^{2} d t, & \left\|\widetilde{\varepsilon}_{N}\right\|^{2}=\int_{I}\left|y(t)-\widetilde{y}_{N}(t)\right|^{2} d t,
\end{array}
$$

if the exact solution is available, and in case we do not know the exact solutions $x(t)$ and $y(t)$, the errors are estimated by

$$
\begin{array}{rlrl}
\left\|e_{N}^{e s}\right\|^{2} & =\int_{I}\left|x_{N}(t)-x_{2 N}(t)\right|^{2} d t, & & \left\|\widetilde{e}_{N}^{e s}\right\|^{2}=\int_{I}\left|\widetilde{x}_{N}(t)-\widetilde{x}_{2 N}(t)\right|^{2} d t, \\
\left\|\varepsilon_{N}^{e s}\right\|^{2} & =\int_{I}\left|y_{N}(t)-y_{2 N}(t)\right|^{2} d t, & \left\|\widetilde{\varepsilon}_{N}^{e s}\right\|^{2}=\int_{I}\left|\widetilde{y}_{N}(t)-\widetilde{y}_{2 N}(t)\right|^{2} d t .
\end{array}
$$

The orders of convergence are calculated by

$$
\begin{array}{ll}
\operatorname{order}_{e}(N) \simeq\left|\log _{2} \frac{\left\|e_{2 N}\right\|}{\left\|e_{N}\right\|}\right|, & \operatorname{order}_{\varepsilon}(N) \simeq\left|\log _{2} \frac{\left\|\varepsilon_{2 N}\right\|}{\left\|\varepsilon_{N}\right\|}\right|, \\
\operatorname{order}_{\tilde{e}}(N) \simeq\left|\log _{2} \frac{\left\|\widetilde{e}_{2 N}\right\|}{\left\|\widetilde{e}_{N}\right\|}\right|, & \operatorname{order}_{\tilde{\varepsilon}}(N) \simeq\left|\log _{2} \frac{\left\|\widetilde{\varepsilon}_{2 N}\right\|}{\left\|\widetilde{\varepsilon}_{N}\right\|}\right|,
\end{array}
$$

EXAMPLE 5.1. Consider the fractional-order differential algebraic equation

$$
\left\{\begin{aligned}
D^{\frac{1}{2}} x(t) & =-x(t)+y(t)+q_{1}(t), \\
0 & =x(t)+y(t)+q_{2}(t), \\
x(0) & =y(0)=0, \quad t \in I,
\end{aligned}\right.
$$

where $q_{1}(t), q_{2}(t)$ are determined by the condition that the exact solutions are given by

$$
x(t)=e^{-\sqrt{t}}-1, \quad y(t)=\sin (\sqrt{t}) .
$$

The regularity of the exact solutions coincides with (2.2) with $\tilde{v}=0$. Here, we illustrate the effect of the proposed regularization process for obtaining the highly accurate approximation to this non-smooth problem and confirm the obtained theoretical results of Theorems 3.6 and 4.1. The asymptotic behavior of the functions $q_{1}(t)$ and $q_{2}(t)$ is given by

$$
\begin{aligned}
& q_{1}(t)=-\frac{\sqrt{\pi}}{2}+\left(-2+\frac{1}{\sqrt{\pi}}\right) \sqrt{t}+O(t), \\
& q_{2}(t)=-\frac{t}{2}+\frac{t \sqrt{t}}{3}+O\left(t^{2}\right),
\end{aligned}
$$

so we have $q_{1}(t) \in B^{1}(I)$ and $q_{2}(t) \in B^{3}(I)$. Therefore, by Theorem 3.6 we can expect that $x_{N}(t)$ and $y_{N}(t)$ approximate the solutions of (5.1) with a linear rate of convergence $\left(k_{1}=1\right.$, $\left.k_{2}=3\right)$. 


\section{ETNA}

Kent State University and

Johann Radon Institute (RICAM)

TABLE 5.1

The obtained errors for Example 5.1 before regularization.

\begin{tabular}{ccccc}
$\mathrm{N}$ & $\left\|e_{N}\right\|$ & $\left\|\varepsilon_{N}\right\|$ & $\operatorname{order}_{e}(N)$ & $\operatorname{order}_{\varepsilon}(N)$ \\
\hline 2 & $2.1462 \times 10^{-1}$ & $2.2174 \times 10^{-1}$ & 0.5421 & 0.5762 \\
4 & $1.4743 \times 10^{-1}$ & $1.4873 \times 10^{-1}$ & 0.5985 & 0.6082 \\
8 & $9.7353 \times 10^{-2}$ & $9.7572 \times 10^{-2}$ & 0.6022 & 0.6776 \\
16 & $6.4130 \times 10^{-2}$ & $6.1023 \times 10^{-2}$ & 0.6734 & 0.7171 \\
32 & $4.0213 \times 10^{-2}$ & $3.7124 \times 10^{-2}$ & 0.8900 & 0.8778
\end{tabular}

TABLE 5.2

The obtained errors for Example 5.1 after regularization.

\begin{tabular}{ccccc}
$\mathrm{N}$ & $\left\|\widetilde{e}_{N}\right\|$ & $\left\|\widetilde{\varepsilon}_{N}\right\|$ & $\operatorname{order}_{\tilde{e}}(N)$ & $\operatorname{order}_{\tilde{\varepsilon}}(N)$ \\
\hline 2 & $9.085 \times 10^{-3}$ & $6.436 \times 10^{-3}$ & 10.8 & 6.73 \\
4 & $5.11 \times 10^{-5}$ & $6.067 \times 10^{-5}$ & 15.32 & 18.72 \\
8 & $1.253 \times 10^{-10}$ & $1.403 \times 10^{-10}$ & 19.22 & 20.26 \\
16 & $2.05 \times 10^{-16}$ & $1.118 \times 10^{-16}$ & 1.18 & 1.79 \\
32 & $4.66 \times 10^{-16}$ & $3.88 \times 10^{-16}$ & 0.801 & 0.536
\end{tabular}

Now, we solve (5.1) using the proposed Legendre collocation method in Section 3 and report the values of $\left\|e_{N}\right\|$ and $\left\|\varepsilon_{N}\right\|$ along with its estimated convergence orders versus the approximation degree $N$ in Table 5.1. As can be seen, the estimated orders $\operatorname{order}_{e}(N)$ and $\operatorname{order}_{\varepsilon}(N)$ tend to 1 as the approximation degree $N$ tends to infinity, and this confirms the linear order of convergence that is predicted by Theorem 3.6.

To calculate a high-order approximate solution, following the method described in Section 4, we employ the coordinate transformation

$$
t=u^{2}, \quad u=\sqrt{t}, \quad s=w^{2}, \quad w=\sqrt{s},
$$

and obtain the regularized equation

$$
\begin{array}{r}
\bar{D}^{\alpha} \bar{x}(u)=-\bar{x}(u)+\bar{y}(u)+\bar{q}_{1}(u), \\
0=\bar{x}(u)+\bar{y}(u)+\bar{q}_{2}(u),
\end{array}
$$

with $\bar{x}(0)=\bar{y}(0)=0$ and $\bar{x}(u)=e^{-u}-1, \bar{y}(u)=\sin (u)$ as infinitely smooth exact solutions. Trivially, the functions $\bar{q}_{i}(u)=q_{i}\left(t^{2}\right), i=1,2$, are infinitely smooth $\left(k_{1}=k_{2}=\infty\right.$ in Theorem 4.1), and thereby an exponential rate of convergence can be expected by implementing the Legendre collocation method to approximate the solutions of (5.2).

The obtained numerical results from the Legendre collocation method for (5.2) are given in Table 5.2. Comparing the results listed in Tables 5.1 and 5.2, we can observe a significant growth in accuracy after utilizing the smoothing procedure. It is noticed that the order reduction for $N=16$ and $N=32$ in Table 5.2 is due to the fact that the obtained numerical errors are very close to machine precision when $16 \leq N \leq 64$ such that in this case we have $\left\|\widetilde{e}_{N}\right\| \simeq c_{e} \times 10^{-16}$ with $1 \leq c_{e} \leq 5$ and $\left\|\widetilde{\varepsilon}_{N}\right\| \simeq c_{\varepsilon} \times 10^{-16}$ with $1 \leq c_{\varepsilon} \leq 6$.

Also, we plot the obtained numerical errors in terms of the number $N$, the degree of approximation, before and after applying the regularization process in Figure 5.1. Indeed, this figure indicates that after applying the smoothing procedure, the predicted exponential rate of convergence in Theorem 4.1 is obtained because in this semi-log diagram, the error varies almost linear versus the degree of approximation, while before regularization, we have very poor convergence results. 

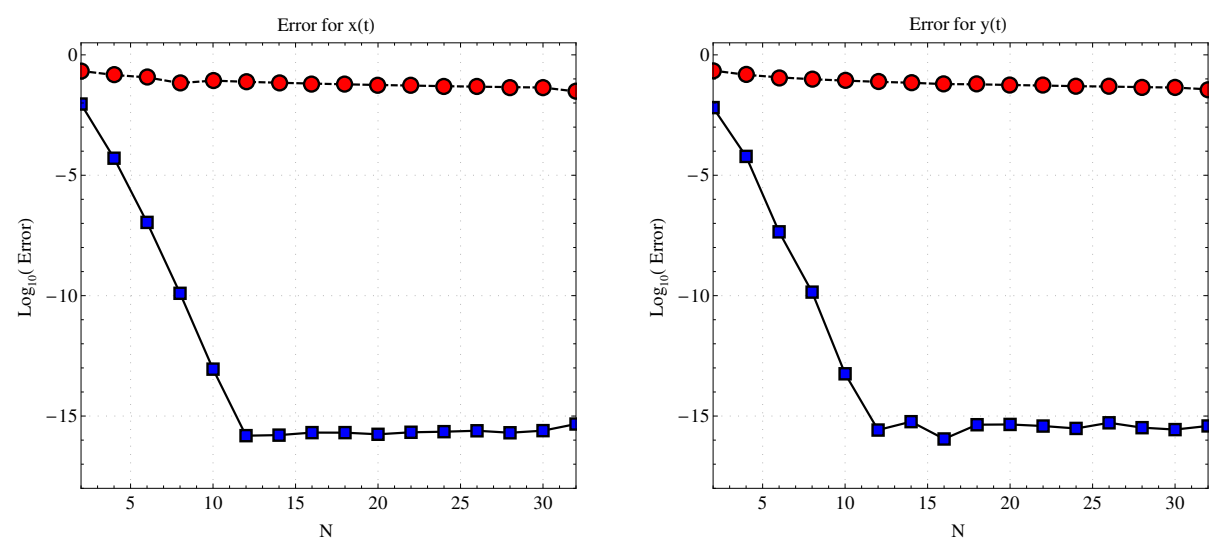

FIG. 5.1. Comparison of the rate of convergence for Example 5.1 before (dashed lines) and after (solid lines) regularization.

EXAMPLE 5.2. Consider the following fractional-order differential algebraic equation

$$
\left\{\begin{aligned}
D^{\frac{1}{4}} x(t) & =e^{t} x(t)+t y(t)+q_{1}(t), \\
0 & =t^{2} x(t)+y(t)+q_{2}(t), \\
x(0) & =y(0)=0, \quad t \in I
\end{aligned}\right.
$$

with the solutions

$$
x(t)=\sinh \sqrt{t}, \quad y(t)=\tan \sqrt{t} .
$$

In this problem, the asymptotic behavior of $q_{1}(t), q_{2}(t)$ is given by

$$
\begin{aligned}
& q_{1}(t)=\frac{\sqrt{\pi}}{2 \Gamma\left(\frac{5}{4}\right)} x^{\frac{1}{4}}-\sqrt{t}+O\left(t^{\frac{5}{4}}\right), \\
& q_{2}(t)=-\sqrt{t}-\frac{t \sqrt{t}}{3}+O\left(t^{\frac{5}{4}}\right),
\end{aligned}
$$

so we have $q_{1}(t), q_{2}(t) \in B^{1}(I)$ (i.e., $k_{1}=k_{2}=1$ in Theorem 3.6), and the Legendre collocation approximation of (5.3) has a linear rate of convergence. Approximation results for an implementation of the proposed scheme in Section 3 for approximating (5.3) are presented in Table 5.3. They confirm the linear order of convergence predicted by Theorem 3.6.

Now, to discover the desired exponential rate of convergence, we proceed with the strategy proposed in Section 4 by applying the variable transformation

$$
t=u^{4}, \quad u=\sqrt[4]{t}, \quad s=w^{4}, \quad w=\sqrt[4]{s},
$$

to obtain the regularized problem with infinitely smooth given functions $\bar{q}_{1}(u), \bar{q}_{2}(u)$ and exact solutions $\bar{x}(u)=\sinh \left(u^{2}\right), \bar{y}(u)=\tan \left(u^{2}\right)$. Thus, we expect an exponential rate of convergence for the Legendre collocation solutions of (5.3) $\left(k_{1}=k_{2}=\infty\right.$ in Theorem 4.1).

Now, we apply the Legendre collocation scheme described in Section 4 and report the obtained numerical results in Table 5.4. As we can see, the rate of convergence increases after regularization in comparison with the given results before regularization in Table 5.3. Again, the order reduction for $N=16,32$ is due to a closeness of the errors to machine precision. 


\section{ETNA}

Kent State University and

Johann Radon Institute (RICAM)

TABLE 5.3

The obtained errors for Example 5.2 before regularization.

\begin{tabular}{ccccc}
$\mathrm{N}$ & $\left\|e_{N}\right\|$ & $\left\|\varepsilon_{N}\right\|$ & $\operatorname{order}_{e}(N)$ & $\operatorname{order}_{\varepsilon}(N)$ \\
\hline 2 & $1.0659 \times 10^{-1}$ & $2.3267 \times 10^{-1}$ & 0.4968 & 0.5924 \\
4 & $7.5538 \times 10^{-2}$ & $1.5273 \times 10^{-1}$ & 0.8675 & 1.1483 \\
8 & $1.3781 \times 10^{-1}$ & $6.8905 \times 10^{-2}$ & 1.0898 & 0.6789 \\
16 & $6.4749 \times 10^{-2}$ & $4.3042 \times 10^{-2}$ & 0.7977 & 0.7834 \\
32 & $3.7247 \times 10^{-2}$ & $2.5007 \times 10^{-2}$ & 0.8799 & 0.8875
\end{tabular}

TABLE 5.4

The obtained errors for Example 5.2 after regularization.

\begin{tabular}{ccccc}
$\mathrm{N}$ & $\left\|\widetilde{e}_{N}\right\|$ & $\left\|\widetilde{\varepsilon}_{N}\right\|$ & $\operatorname{order}_{\tilde{e}}(N)$ & $\operatorname{order}_{\tilde{\varepsilon}}(N)$ \\
\hline 2 & $1.11 \times 10^{-2}$ & $5.694 \times 10^{-2}$ & 1.28 & 2.095 \\
4 & $4.576 \times 10^{-3}$ & $1.333 \times 10^{-2}$ & 9.68 & 5.347 \\
8 & $5.629 \times 10^{-6}$ & $3.276 \times 10^{-4}$ & 24.72 & 10.5 \\
16 & $2.044 \times 10^{-13}$ & $2.263 \times 10^{-7}$ & 9.98 & 21.63 \\
32 & $2.029 \times 10^{-16}$ & $6.988 \times 10^{-14}$ & 0.1601 & 8.28
\end{tabular}

Also, in Figure 5.2, we compare the corresponding semi-log errors of the approximate solutions versus $N$ before and after applying the regularization process to monitor the convergence behavior of the solutions. The predicted exponential rate of convergence is confirmed by Figure 5.2 because in this semi-log diagram, the errors varies almost linearly versus $N$ after applying the regularization process.

EXAMPLE 5.3 ([6]). Consider the fractional-order differential algebraic equation

$$
\left\{\begin{aligned}
D^{\alpha} x(t) & =-x(t)+y(t)-\sin t, \\
0 & =x(t)+y(t)-e^{-t}-\sin t, \\
x(0) & =1, \quad y(0)=0, \quad t \in I,
\end{aligned}\right.
$$

where $0<\alpha \leq 1$ and the exact solutions are given by $x(t)=e^{-t}, y(t)=\sin t$ when $\alpha=1$.

The numerical errors obtained from an implementation of the described scheme with and without applying the regularization process are reported in Tables 5.5 and 5.6. Indeed, these results confirm the effectiveness of the regularization procedure by an increase in the accuracy of the approximate solutions when we do not have access to the exact solutions.

In addition, Figure 5.3 displays the approximate solutions of Example 5.3 obtained for different values of $\alpha$ and $N=16$ using the proposed method with the regularization strategy. The results indicate that as $\alpha$ tends to 1 , the approximate solutions converge to the solutions obtained for $\alpha=1$.

TABLE 5.5

The obtained errors for Example 5.3 with different values of $\alpha$ before regularization.

\begin{tabular}{|c|c|c|c|c|c|c|}
\hline \multirow[b]{2}{*}{$N$} & \multicolumn{3}{|c|}{$\left\|e_{N}^{e s}\right\|$} & \multicolumn{3}{|c|}{$\left\|\varepsilon_{N}^{e s}\right\|$} \\
\hline & $\alpha=0.2$ & $\alpha=0.6$ & $\alpha=0.8$ & $\alpha=0.2$ & $\alpha=0.6$ & $\alpha=0.8$ \\
\hline 4 & $3.8 \times 10^{-2}$ & $1.84 \times 10^{-2}$ & $9.31 \times 10^{-3}$ & $3.79 \times 10^{-2}$ & $1.83 \times 10^{-2}$ & $9.3 \times 10^{-3}$ \\
\hline 8 & $2.07 \times 10^{-2}$ & $6.77 \times 10^{-3}$ & $2.81 \times 10^{-3}$ & $2.06 \times 10^{-2}$ & $6.76 \times 10^{-3}$ & $2.8 \times 10^{-3}$ \\
\hline 16 & $1.07 \times 10^{-2}$ & $2.15 \times 10^{-3}$ & $8.15 \times 10^{-4}$ & $1.06 \times 10^{-2}$ & $2.14 \times 10^{-3}$ & $8.1 \times 10^{-4}$ \\
\hline 32 & $2.08 \times 10^{-3}$ & $2.95 \times 10^{-4}$ & $1.06 \times 10^{-4}$ & $2.07 \times 10^{-3}$ & $2.85 \times 10^{-4}$ & $1.05 \times 10^{-4}$ \\
\hline
\end{tabular}



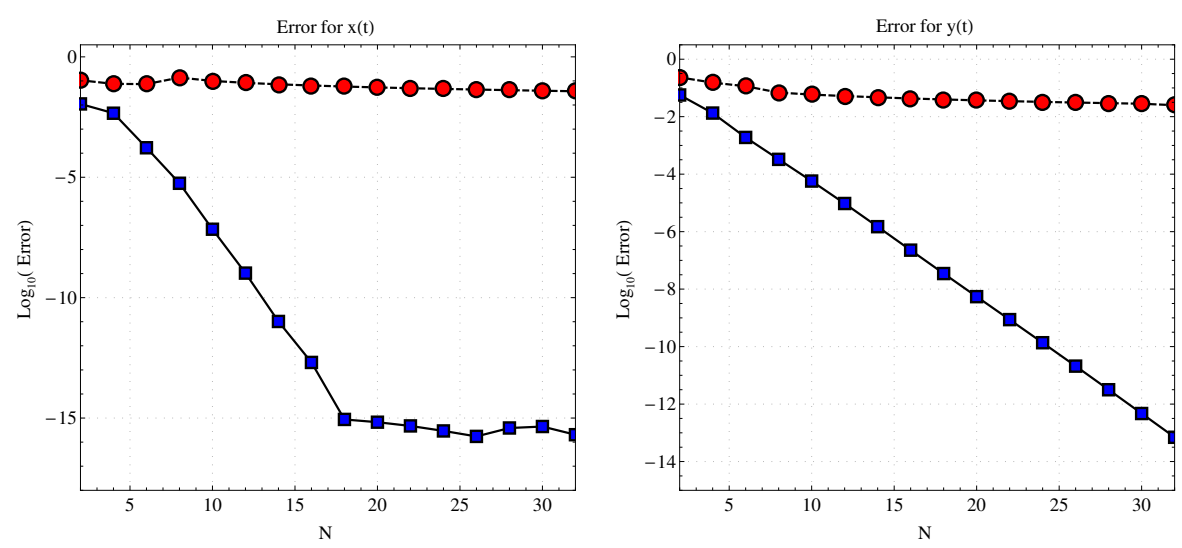

FIG. 5.2. Comparison of the rate of convergence for Example 5.2 before (dashed lines) and after (solid lines) regularization.

TABLE 5.6

The obtained errors for Example 5.3 with different values of $\alpha$ after regularization.

\begin{tabular}{ccccccc} 
& \multicolumn{5}{c}{$\left\|\widetilde{e}_{N}^{e s}\right\|$} \\
$N$ & $\alpha=0.2$ & $\alpha=0.6$ & $\alpha=0.8$ & $\alpha=0.2$ & $\alpha=0.6$ & $\alpha=0.8$ \\
\hline 4 & $9.49 \times 10^{-4}$ & $2.43 \times 10^{-3}$ & $5.84 \times 10^{-3}$ & $3.15 \times 10^{-3}$ & $4.38 \times 10^{-3}$ & $5.59 \times 10^{-3}$ \\
8 & $1.55 \times 10^{-5}$ & $6.65 \times 10^{-5}$ & $7.85 \times 10^{-5}$ & $9.78 \times 10^{-5}$ & $8.66 \times 10^{-5}$ & $1.53 \times 10^{-4}$ \\
16 & $2.09 \times 10^{-10}$ & $1.13 \times 10^{-9}$ & $1.91 \times 10^{-8}$ & $1.05 \times 10^{-9}$ & $1.78 \times 10^{-9}$ & $1.94 \times 10^{-8}$ \\
32 & $4.14 \times 10^{-16}$ & $4.01 \times 10^{-16}$ & $9.66 \times 10^{-16}$ & $8.89 \times 10^{-16}$ & $4.84 \times 10^{-16}$ & $9.61 \times 10^{-16}$
\end{tabular}

EXAMPLE 5.4. Consider the fractional-order differential algebraic equation

$$
\left\{\begin{aligned}
D^{\alpha} x(t) & =x(t)+2 y(t)+q_{1}(t), \\
0 & =2 x(t)+y(t)+q_{2}(t), \\
x(0) & =y(0)=0, \quad t \in[0,2 \pi],
\end{aligned}\right.
$$

where $q_{1}(t)$ and $q_{2}(t)$ are chosen such that the exact solutions are $x(t)=\sqrt{t} \sin \left(t^{2}\right)$ and $y(t)=\sqrt{t} \cos \left(t^{2}\right)$.

The numerical results for applying the proposed scheme to Example 5.4 with and without the regularization procedure are reported in Table 5.7 and Figure 5.4. Due to the oscillatory behavior of the exact solution and the long time integration domain, the approximate solutions are in a good agreement with the exact ones only for large values of $N$ after applying the regularization process.

6. Conclusion. In this paper, we investigate the numerical behavior of the Legendre collocation technique to obtain an approximation solution for the linear semi-explicit fractional differential algebraic equation (1.1). We proved that the derivatives of the exact solution behave like $t^{\alpha-1}$ near the origin, and this degrades the accuracy of the approximate solution. To resolve this problem, we change the underlying equation into a new equation with improved regularity properties employing a variable transformation according to the asymptotic performance of the unknown solution. Theoretical predictions along with the numerical examples indicate a meaningful improvement in the accuracy after applying the smoothing procedure. 


\section{ETNA}

Kent State University and Johann Radon Institute (RICAM)
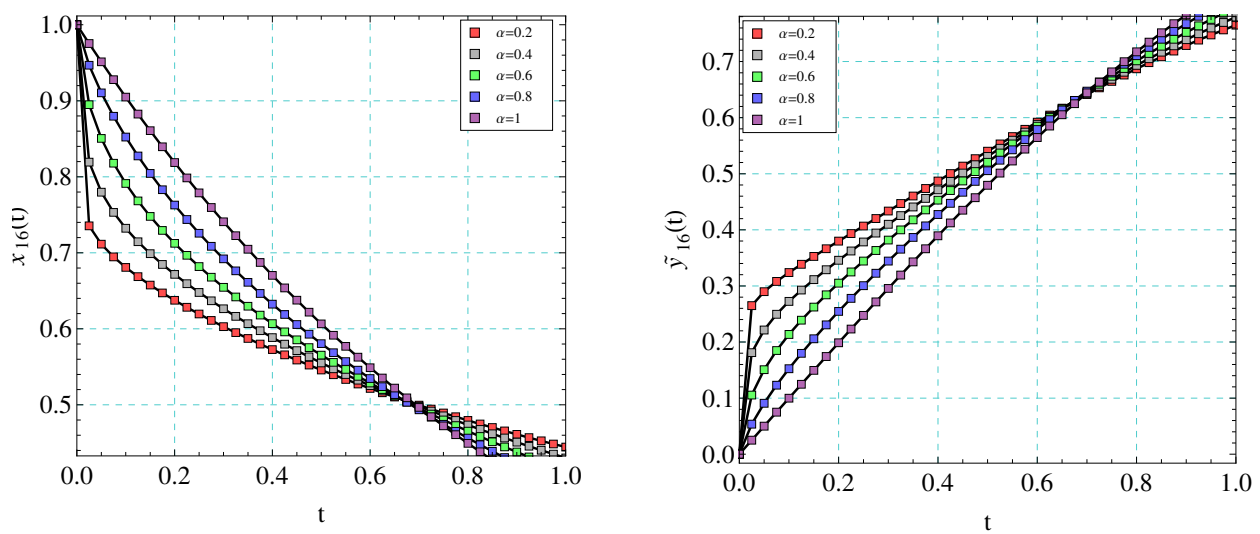

FIG. 5.3. The approximated solutions of Example 5.3 for various values of $\alpha$ and $N=16$.

TABLE 5.7

The obtained errors of Example 5.4 with different values of $\alpha$ before and after regularization.

Before regularization

\begin{tabular}{ccccc}
$N$ & $\left\|e_{N}\right\|$ & $\left\|\varepsilon_{N}\right\|$ & $\left\|\widetilde{e}_{N}\right\|$ & $\left\|\widetilde{\varepsilon}_{N}\right\|$ \\
\hline 30 & $2.63 \times 10^{-1}$ & $5.17 \times 10^{-1}$ & $8.04 \times 10^{-1}$ & $9.58 \times 10^{-1}$ \\
40 & $2.52 \times 10^{-1}$ & $5.03 \times 10^{-1}$ & $8.98 \times 10^{-2}$ & $1.45 \times 10^{-1}$ \\
50 & $1.99 \times 10^{-1}$ & $3.72 \times 10^{-1}$ & $1.09 \times 10^{-3}$ & $1.94 \times 10^{-3}$ \\
60 & $1.81 \times 10^{-1}$ & $3.6 \times 10^{-1}$ & $1.99 \times 10^{-6}$ & $3.84 \times 10^{-6}$ \\
70 & $1.79 \times 10^{-1}$ & $3.51 \times 10^{-1}$ & $4.64 \times 10^{-9}$ & $7.82 \times 10^{-9}$
\end{tabular}

\section{REFERENCES}

[1] K. E. Brenan, S. L. CAmpbell, And L. R. Petzold, Numerical Solution of Initial-Value Problems in Differential-Algebraic Equations, SIAM, Philadelphia, 1996.

[2] Y. CHEN AND T. TANG, Convergence analysis of the Jacobi spectral collocation methods for Volterra integral equations with a weakly singular kernel, Math. Comp., 79 (2010), pp. 147-167.

[3] S. K. DAMARLa AND M. KUnDU, Numerical solution of fractional order differential algebraic equations using generalized triangular function operational matrices, Fract. Calc. Appl., 6, (2015), pp. 31-35

[4] K. Diethelm, The Analysis of Fractional Differential Equations, Springer, Berlin, 2010.

[5] X.-L. DING AND Y.-L. JiANG, Waveform relaxation method for fractional differential algebraic equations, Fract. Calc. Appl. Anal., 17 (2014), pp. 585-604.

[6] B. İBIS AND M. BAYRAM, Numerical comparison of methods for solving fractional differential-algebraic equations (FDAEs), Comput. Math. Appl., 62 (2011), pp. 3270-3278.

[7] B. İBIS, M. BAYRAM AND A. GÖKSEL AĞARGÜN, Applications of fractional differential transform method to fractional differential-algebraic equations, Eur. J. Pure Appl. Math., 4 (2011), pp. 129-141.

[8] H. M. Jaradat, M. Zurigat, AND S. Al-Shara, Toward a new algorithm for systems of fractional differential algebraic equations, Ital. J. Pure. Appl. Math., 32 (2014), pp. 579-594.

[9] P. MOKHTARY, Reconstruction of exponentially rate of convergence to Legendre collocation solution of a class of fractional integro-differential equations, J. Comput. Appl. Math., 279 (2015), pp. 145-158.

[10] P. MOKHTARY AND F. GHOREISHI, Convergence analysis of the operational Tau method for Abel-type Volterra integral equations, Electron. Trans. Numer. Anal., 41 (2014), pp. 289-305. http://etna.ricam.oeaw.ac.at/vol.41.2014/pp289-305.dir/pp289-305.pdf

[11] E. ORTIZ AND H. SAMARA, An operational approach to the Tau method for the numerical solution of nonlinear differential equations, Computing, 27 (1981), pp. 15-25.

[12] I. Podlubny, Fractional Differential Equations, Academic Press, San Diego, 1999.

[13] T. J. Rivlin, An Introduction to the Approximation of Functions, Dover, New York, 1969.

[14] W. Rudin, Principles of Mathematical Analysis, McGraw-Hill, New York, 1976.

[15] J. Shen, T. TANG, And L.-L. WANG, Spectral Methods Algorithms, Analysis and Applications, Springer, Heidelberg, 2011. 

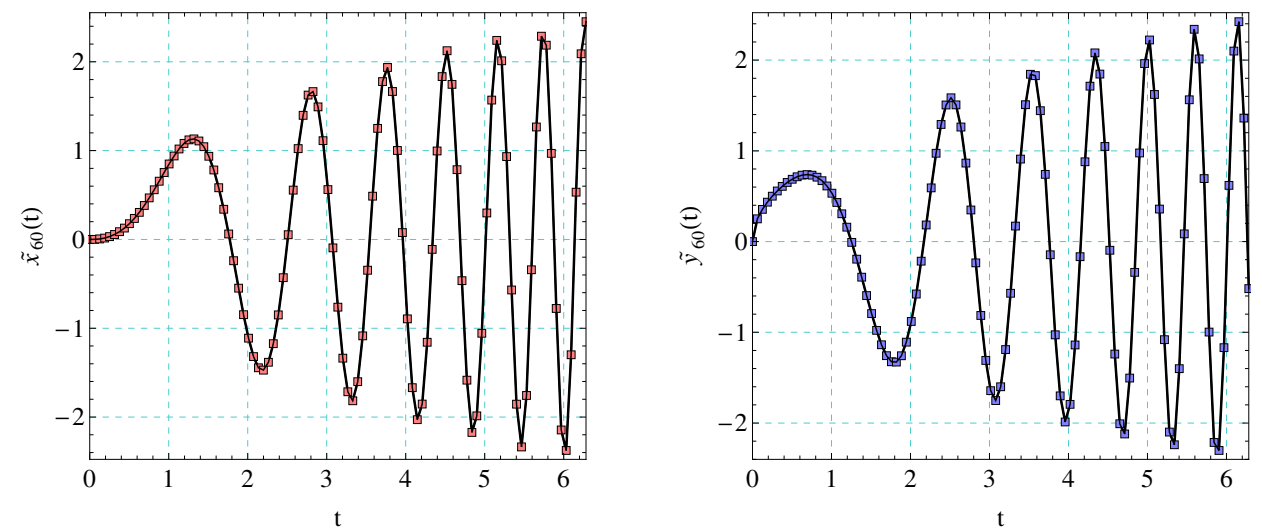

FIG. 5.4. Plots of the approximate solutions (rectangle markers) and the exact solutions (solid lines) of $x(t)$ (left-hand side) and $y(t)$ (right-hand side) using the regularization process and $N=60$ for Example 5.4.

[16] H.-S. WANG, W.-L. JHU, C.-F. Yung, AND P.-F. WANG, Numerical solutions of differential algebraic equations and its applications in solving TPPC problems, J. Mar. Sci. Technol., 19 (2011), pp. 76-88.

[17] S. Westerlund And L. Ekstam, Capacitor theory, IEEE Trans. Dielectr. Electr. Insul., 1 (1994), pp. 826839.

[18] S. Westerlund, Dead Matter Has Memory!, Casual Consulting, Kalmar, 2002.

[19] M. Zurigat, S. Momani, AND A. Alawneha, Analytical approximate solutions of systems of fractional algebraic differential equations by homotopy analysis method, Comput. Math. Appl., 59 (2010), pp. 12271235. 